\title{
Thyroglobulin Wash Testing in the Surveillance of Patients with Thyroid Carcinoma: Proposal for a Reflex Test
}

\author{
Anastasiya Pigala $^{a}$ Rossitza Draganova-Tacheva ${ }^{a, b}$ \\ Charalambos C. Solomides ${ }^{a, b} \quad$ Marluce Bibbo ${ }^{a, b}$ \\ aThomas Jefferson University Hospital, and b Department of Pathology, Anatomy and Cell Biology, Jefferson Medical \\ College, Philadelphia, Pa., USA
}

\section{Key Words}

Thyroid carcinoma · Fine needle aspiration - Thyroglobulin . Lymph node $\cdot$ Reflex test $\cdot$ Thyroid bed

\begin{abstract}
Objective: Fine needle aspiration (FNA) cytology with thyroglobulin wash (TG-W) testing is recommended for follow-up of patients with differentiated thyroid carcinoma (DTC). The goal of this retrospective study was to determine if TG-W results contributed to the management of cases with positive FNA cytology. Study Design: We reviewed data on patients with positive and suspicious cytology results, undergoing lymph node or thyroid bed FNA with TG-W testing as part of the preoperative or follow-up investigation of histologically proven DTC in our institution and from the literature. $\boldsymbol{R e}$ sults: Of 30 positive/suspicious lymph node and thyroid bed FNAs in our institution, 22 (73\%) had an elevated (>1 ng/ml) TG-W level. Seven of 8 TG-W-negative cases had DTC on follow-up. Of 577 cytology-positive/suspicious FNAs in the literature, 557 (97\%) showed TG-W-positive results. Fourteen of $20 \mathrm{TG}-\mathrm{W}$-negative cases had DTC on follow-up. All patients in retrospective and literature review groups with positive and suspicious FNA cytology and available follow-up were treated for recurrent or metastatic disease regardless
\end{abstract}

of TG-W results. Conclusion: Observations of both our and other institutions support a recommendation of reflex FNA TG-W testing only for cases with negative or indeterminate cytology results.

(c) 2013 S. Karger AG, Basel

\section{Introduction}

The standard investigation of a patient with suspected differentiated thyroid carcinoma (DTC) is an ultrasound study with fine needle aspiration (FNA). This approach has been recommended for both follow-up after thyroidectomy and the investigation of suspicious cervical lymph nodes prior to the surgery. Multiple studies showed that the addition of simultaneous thyroglobulin testing of FNA needle wash fluid (TG-W) to cytology improved the sensitivity for disease detection. These results were reflected in the recommendations of the American Thyroid Association which included both ultrasound-guided FNA cytology and TG-W testing of suspicious nodes in the follow-up for DTC [1].

Our study was aimed to assess the value of TG-W measurement in a cohort of cytology-positive patients. Analysis included 30 cytology-positive and -suspicious FNAs

\section{KARGER}

E-Mail karger@karger.com

www.karger.com/acy
(C) 2013 S. Karger AG, Basel

0001-5547/13/0576-0545\$38.00/0
Correspondence to: Dr. Anastasiya Pigal

Thomas Jefferson University Hospital

132 South 10th Street

Philadelphia, PA 19107 (USA)

E-Mail anastasia_pigal@hotmail.com 
from our institution and 577 cases described in the literature. We hypothesized that TG-W testing can be safely omitted in patients with positive and suspicious FNA cytology, thus supporting a reflex testing approach.

\section{Methods}

For this retrospective study, all cases with simultaneous FNA cytology and TG-W testing performed at Thomas Jefferson University Hospital over a period of 2 years were selected. The group consisted of FNAs with positive or suspicious diagnoses in cytology and available TG-W results for samples collected at the time of FNA. Only cases with a history of or subsequently histologically proven DTC were included into the study. The study was approved by the institutional review board.

Ultrasound-guided FNA of neck lymph nodes with suspicious features and thyroid bed nodules was performed by experienced radiologists using 25- to 27-gauge needles. After a few passes for cytology, the operator dedicated one pass for TG-W testing. The needle was washed in $1 \mathrm{ml}$ of saline solution. The sample was then refrigerated before being sent to an outside laboratory for testing.

An extensive literature review using internet search engines resulted in a number of articles describing DTC cases with positive and suspicious FNA cytology and corresponding TG-W levels. These cases composed our literature review group. Cases with a histological follow-up diagnosis of malignancy other than DTC were excluded from analysis. TG-W testing in literature cases was performed on washout fluid from the needle used for FNA of the nodule. The collection technique slightly varied among the institutions. Most investigators in the reviewed articles described rinsing the needle in $0.5-1 \mathrm{ml}$ of saline or specialized thyroglobulin-free solution after obtaining the material for cytology slides from one or more passes.

\section{Results}

Our retrospective study included 30 FNAs with positive/suspicious cytology, of which 23 (77\%) were lymph nodes (10 preoperative and 13 thyroidectomized) and 7 thyroid bed nodules (fig. 1). A total of 22 FNAs (73\%) had an elevated ( $>1 \mathrm{ng} / \mathrm{ml})$ TG-W level, with a range of 55$53,835 \mathrm{ng} / \mathrm{ml}$. The remaining 8 cases of FNA with negative TG-W $(<1 \mathrm{ng} / \mathrm{ml})$ were treated for recurrent/metastatic disease. Five of them were subsequently proven to be DTC on histological examination, 1 case had no evidence of DTC in the resected material, and 2 cases were treated conservatively for recurrence.

Review of published studies resulted in a group of 577 FNAs with positive/suspicious cytology and simultaneous TG-W testing, including 548 lymph nodes and 29 thyroid bed nodules. The majority of the literature review cases with positive or suspicious FNA cytology had surgi-

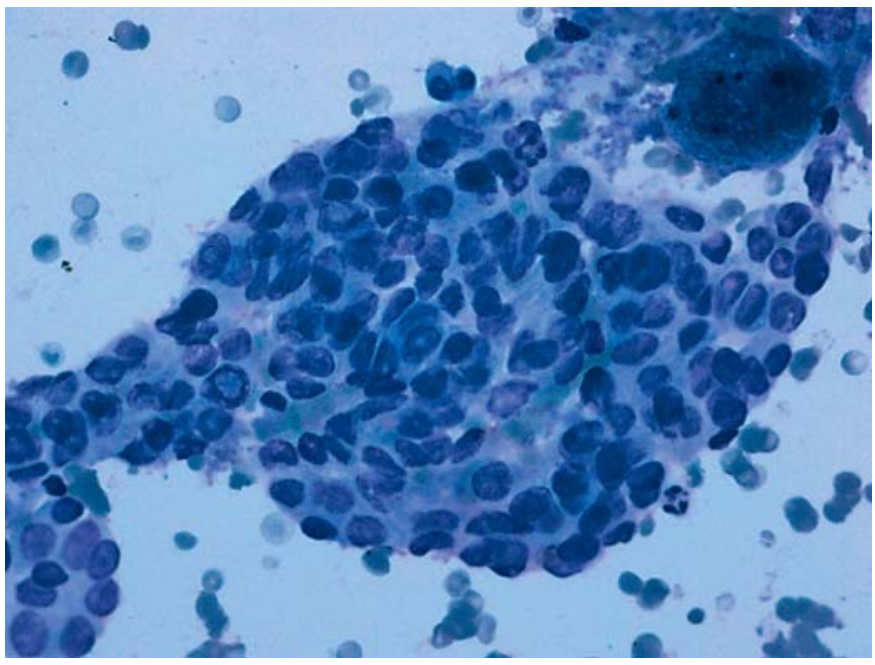

Fig. 1. Thyroid bed nodule FNA smear showing clusters of follicular cells with nuclear enlargement, nuclear groves and intranuclear pseudoinclusions, diagnostic of papillary thyroid carcinoma. The simultaneous TG-W test result was positive. Diff-Quik. $\times 600$.

cal follow-up. A total of 557 FNAs (97\%) showed TG-Wpositive results at the corresponding study cutoff levels. Of 20 TG-W-negative FNAs, 14 had DTC on histological follow-up, 5 were negative and 1 was lost to follow-up. TG-W result concordance with positive/suspicious cytology varied from 75 to $100 \%$ in the reviewed studies (table 1). The follow-up on cases with discrepant FNA cytology and TG-W results is summarized in table 2.

\section{Discussion}

Ultrasound-guided FNA cytology with simultaneous TG-W testing is a standard modality for the follow-up of patients with DTC after thyroidectomy. Positive FNA cytology is regarded as reliable evidence of recurrent disease. It is used for both lymph nodes and thyroid bed nodules. The recommendations to add TG-W testing to FNA cytology are based on strong evidence of increased sensitivity of combined testing over cytology alone $[2,6,8,10$, $12,14]$. Positive FNA cytology in cervical lymph nodes at the time of initial investigation for DTC will necessitate the extension of the surgery to include the involved group of lymph nodes [1]. Despite the wide use of this diagnostic modality, no one has assessed the utility of TG-W testing in patients with positive and suspicious cytology.

Excellent diagnostic accuracy of FNA TG-W reflected in several studies resulted in proposals to substitute FNA 
Table 1. TG-W test results in cases with positive and suspicious FNA cytology in reviewed studies

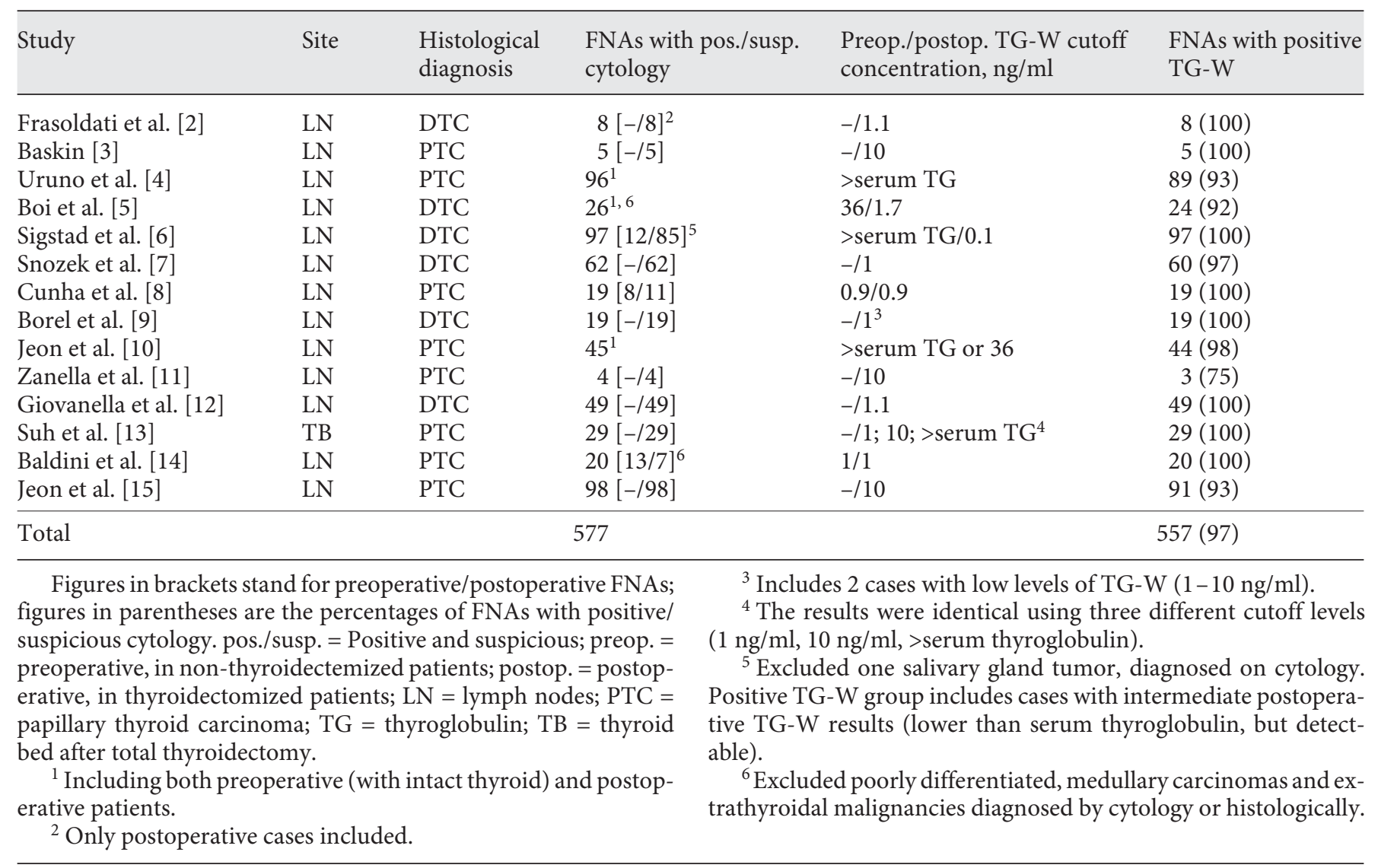

cytology with the TG-W test [7]. On the other hand, some authors commented on the value of cytology in poorly differentiated, anaplastic and medullary carcinomas, residual normal thyroid tissue and metastatic malignancies from other organs $[5,13]$. Furthermore, residual thyroid tissue nodules after thyroidectomy without radioablation, a potential source of elevated TG-W levels in the absence of carcinoma, need to be considered in thyroid bed FNA.

The majority of TG-W test results in both retrospective and literature review groups were concordant with positive/suspicious FNA cytology. The results in the retrospective study at our institution show a $27 \%$ rate of TG$\mathrm{W}$-negative results in cytology-positive FNAs, with 7 of 8 discrepant cases representing TG-W false negatives according to follow-up. In the combined literature review group, the rate of discrepant results is $3 \%$, with 14 of 20 being TG-W false negative. The discrepant cases with negative surgical follow-up constituted 3\% of cytologypositive cases in the retrospective group and $1 \%$ in the literature review group.
Table 2. Discordant cases in the literature review group with histological follow-up: FNAs with positive or suspicious cytology and negative TG-W test results

\begin{tabular}{llll}
\hline Study & $\begin{array}{l}\text { FNAs with } \\
\text { pos./susp. } \\
\text { cytology }\end{array}$ & $\begin{array}{l}\text { FNAs with } \\
\text { negative } \\
\text { TG-W }\end{array}$ & $\begin{array}{l}\text { Positive } \\
\text { follow-up } \\
\text { histology }\end{array}$ \\
\hline Uruno et al. [4] & 96 & $7(7)$ & $7(7)$ \\
Boi et al. [5] & 24 & $2(8)$ & 0 \\
Snozek et al. [7] & 62 & $2(3)$ & 0 \\
Jeon et al. [10] & 45 & $1(2)$ & $1(2)$ \\
Zanella et al. [11] & 4 & $1(25)$ & 0 \\
Jeon et al. [15] & 98 & $7(7)^{1}$ & $6(6)$ \\
Total & & $20(3)$ & $14(2)$ \\
\hline
\end{tabular}

Figures in parentheses are the percentages of FNAs with positive/suspicious cytology. pos./susp. = Positive and suspicious.

${ }^{1}$ One FNA follow-up is not available. 
Current practice is to treat recurrent, metastatic or residual DTC with surgery, radioiodine ablation or external beam radiation [1]. A positive/suspicious FNA cytology is considered strong evidence of the disease. Positive TG-W results do not give any additional information to the clinician in these cases. False-negative TG-W results, although infrequently, may potentially influence the patient's judgment in the process of making medical care decisions. In addition, TG-W analysis is often a send-out test requiring substantial time and expense.

There are limitations to our retrospective study. The group size is relatively small because the dedicated TG-W pass could have been skipped whenever diagnosis of DTC became obvious at the time of cytology rapid on site evaluation. The limitation of our literature review case analysis lays in the differences of the TG-W collection technique, testing methods and cutoff levels. The TG-W cutoff levels for lymph nodes varied from 0.9 to $10 \mathrm{ng} / \mathrm{ml}$ in patients after thyroidectomy and from 0.9 to $88.8 \mathrm{ng} / \mathrm{ml}$ (serum thyroglobulin concentration) in patients awaiting thyroidectomy. Authors used different ways to define the TG-W cutoff value: from receiver-operator curve, serum thyroglobulin level or mean +2 SD of TG-W concentration in the control group. Our review does not take into account other possible variables including: presence of anti-thyroglobulin antibodies, sample volume, dilution, operator experience, serum thyroglobulin levels, and technology of thyroglobulin assay. Some of these variables were shown to influence TG-W results at statistically significant levels [15].

In the proposed model of reflex testing, the sample for TG-W is collected at the time of FNA, but TG-W measurement is performed only if the cytology result comes negative or inadequate. The concept of reflex testing has already been in use in cytology for years. Among the tests with a similar workflow is human papilloma virus testing in gynecologic cytology and molecular tests in thyroid FNA. The reflex testing workflow is especially valuable in cost-conscious and limited resource medical practices.

A possible question is related to the cutoff levels for TG-W in patients with preserved contralateral thyroid. Lobectomy is recommended for small low-risk DTC [1]. As a result, a growing population of patients with preserved thyroid function is in need of postoperative follow-up. Borel et al. [9] stated that serum thyroglobulin from FNA sample blood contamination should not significantly influence the FNA TG-W level. Using experiments with albumin and thyroglobulin dilution, they concluded that blood adds only a trivial amount of thyroglobulin to the sample. Further investigation defining TG-W cutoff levels in this population is needed.

\section{Conclusion}

All patients in retrospective and literature review groups with positive and suspicious FNA cytology and available follow-up were treated for recurrent or metastatic disease regardless of TG-W results. Positive FNA cytology has a high positive predictive value and therefore is used as the gold standard. An ability to aid in the diagnosis of poorly differentiated tumors, medullary carcinoma, normal thyroid tissue and extrathyroidal tumors makes it invaluable in preoperative lymph node and thyroid bed FNA. Although simultaneous TG-W testing was shown to improve the sensitivity of FNA cytology in negative and indeterminate cases, its results do not influence the management of cases with positive cytology. Our observations along with the experience of other institutions support the idea of TG-W reflex testing for cases with negative or indeterminate FNA cytology.

\section{References}

$\checkmark 1$ Cooper DS, Doherty GM, Haugen BR, Kloos RT, Lee SL, Mandel SJ, Mazzaferri EL, McIver B, Pacini F, Schlumberger M, Sherman SI, Steward DL, Tuttle RM: Revised American Thyroid Association Management guidelines for patients with thyroid nodules and differentiated thyroid cancer. Thyroid 2009;19: 1167-1214.
2 Frasoldati A, Toschi E, Zini M, Flora M, Caroggio A, Dotti C, Valcavi R: Role of thyroglobulin measurement in fine-needle aspiration biopsies of cervical lymph nodes in patients with differentiated thyroid cancer. Thyroid 1999;9:105-111.

3 Baskin HJ: Detection of recurrent papillary thyroid carcinoma by thyroglobulin assessment in the needle washout after fine-needle aspiration of suspicious lymph nodes. Thyroid 2004;14:959-963.
4 Uruno T, Miyauchi A, Shimizu K, Tomoda C, Takamura Y, Ito Y, Miya A, Kobayashi K, Matsuzuka F, Amino N, Kuma K: Usefulness of thyroglobulin measurement in fine-needle aspiration biopsy specimens for diagnosing cervical lymph node metastasis in patients with papillary thyroid cancer. World J Surg 2005;29:483-485. 
5 Boi F, Baghino G, Atzeni F, Lai ML, Faa G, Mariotti S: The diagnostic value for differentiated thyroid carcinoma metastases of thyroglobulin $(\mathrm{Tg})$ measurement in washout fluid from fine-needle aspiration biopsy of neck lymph nodes is maintained in the presence of circulating anti-Tg antibodies. J Clin Endocrinol Metab 2006;91:1364-1369.

-6 Sigstad E, Heilo A, Paus E, Holgersen K, Groholt KK, Jorgensen LH, Bogsrud TV, Berner A, Bjoro T: The usefulness of detecting thyroglobulin in fine-needle aspirates from patients with neck lesions using a sensitive thyroglobulin assay. Diagn Cytopathol 2007;35:761-767.

7 Snozek CL, Chambers EP, Reading CC, Sebo TJ, Sistrunk JW, Singh RJ, Grebe SKG: Serum thyroglobulin, high-resolution ultrasound, and lymph node thyroglobulin in diagnosis of differentiated thyroid carcinoma nodal metastases. J Clin Endocrinol Metab 2007;92: 4278-4281.
8 Cunha N, Rodrigues F, Curado F, Ilheu O, Cruz C, Naidenov P, Rascao MJ, Ganho J, Gomez I, Pereira H, Real O, Figueiredo P, Campos B, Valido F: Thyroglobulin detection in fine-needle aspirates of cervical lymph nodes: a technique for the diagnosis of metastatic differentiated thyroid cancer. Eur J Endocrinol 2007;157:101-107.

>9 Borel AL, Boizel R, Faure P, Barbe G, Boutonnat J, Sturm N, Segneurin D, Bricault I, Caravel JP, Chaffanjon P, Chabre O: Significance of low levels of thyroglobulin in fine-needle aspirates from cervical lymph nodes of patients with a history of differentiated thyroid cancer. Eur J Endocrinol 2008;158:691-698.

10 Jeon SJ, Kim E, Park JS, Son KR, Baek JH, Kim YS, Park do J, Cho BY, Na DG: Diagnostic benefit of thyroglobulin measurement in fineneedle aspiration for diagnosing metastatic cervical lymph nodes from papillary thyroid cancer: correlations with US features. Korean J Radiol 2009;10:106-111.

11 Zanella AB, Souza Meyer EL, Balzan L, Silva AC, Camargo J, Migliavacca A, Guimaraes JR, Maia AL: Thyroglobulin measurements in washouts of fine needle aspirates in cervical lymph nodes for detection of papillary thyroid cancer metastases. Arq Bras Endocrinol Metabol 2010;54:550-554.
2 Giovanella L, Ceriani L, Suriao S: Lymph node thyroglobulin measurement in diagnosis of neck metastases of differentiated thyroid carcinoma. J Thyroid Res 2011;2011:621839.

13 Suh YJ, Son EJ, Moon HJ, Kim EK, Han KH, Kwak JY: Utility of thyroglobulin measurements in fine-needle aspirates of space occupying lesions in the thyroid bed after thyroid cancer operations. Thyroid 2013;23:280-288.

14 Baldini E, Sorrenti S, Di Gioia C, De Vito C, Antonelli A, Gnessi L, Carbotta G, D'Armiento E, Miccoli P, De Antoni E, Ulisse S: Cervical lymph node metastases from thyroid cancer: does thyroglobulin and calcitonin measurement in fine needle aspirates improve the diagnostic value of cytology? BMC Clin Pathol 2013;13:1-7.

15 Jeon MJ, Park JW, Han JM, Yim JH, Song DE, Gong G, Kim TY, Baek JH, Lee JH, Shong YK, Kim WB: Serum antithyroglobulin antibodies interfere with thyroglobulin detection in fineneedle aspirates of metastatic neck nodes in papillary thyroid carcinoma. J Clin Endocrinol Metab 2013;98:153-160. 